Journal of Applied Biosciences 67:5236 - 5251

ISSN 1997-5902

\title{
Les plantes tinctoriales d'Afrique Centrale : enquête ethnobotanique et screening phytochimique
}

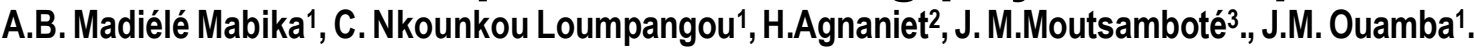

1 Unité de Chimie du Végétal et de la Vie (UC2V), Faculté des Sciences et Techniques, Université Marien Ngouabi, Brazzaville (Congo)

2 Laboratoire des Substances Naturelles et de Synthèse Organométallique (LASUNSO), Faculté des Sciences, Université des Sciences et Techniques de Masuku, Franceville (Gabon)

3 École Nationale Supérieure de l'Agronomie et de la Foresterie (ENSAF), Université Marien Ngouabi Brazzaville (Congo)

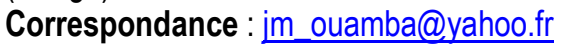

Original submitted in on $3^{\text {rd }}$ May 2013 Published online at www.m.elewa.org on 30 $30^{\text {th }}$ July 2013.

https://dx.doi.org/10.4314/jab.v67i0.95045

\section{Résumé}

Objectif : une étude ethnobotanique des plantes tinctoriales a été menée de juin 2010 à novembre 2011 dans le but de les inventorier et de réaliser un screening chimique sur trois espèces sélectionnées selon un certains nombre de critères.

Méthodologie et résultats : Une enquête ethnobotanique pour les plantes tinctoriales du Congo réalisée de juillet 2010 à novembre 2011 auprès des herboristes, des artisans teinturiers et des détenteurs de savoir traditionnel, et compléter par une revue bibliographique des plantes de la région Afrique Centrale a permis d'inventorier cent cinquante (150) plantes utilisées comme colorants par les populations de la région. Trois de ces plantes, Grewia coriacea Mast (Malvaceae - Grewioideae), Harungana madagascariensis Lam ex Poir. (Hypericaceae), Annickia chlorantha Oliv. (Annonaceae) ont été sélectionnées au regard de leur fréquence d'utilisation pour un screening phytochimique afin d'en évaluer les propriétés tinctoriales. Les flavonoïdes, les tanins, les anthocyanes, les anthraquinones et les alcaloïdes ont été mis évidences.

Conclusion et application : l'inventaire ethnobotanique a permis de nous rendre compte que les plantes tinctoriales sont largement utilisées par les populations à la fois en teinture et en médecine traditionnelle. L'analyse phytochimique des trois espèces sélectionnées présage que celles-ci peuvent être des matières premières pour la production des colorants.

Mots clés : Enquête ethnobotanique, plantes tinctoriales, Grewia coriacea, Harungana madagascariensis, Annickia chlorantha, screening phytochimique.

\section{Abstract}

Objective: An ethnobotanical survey of plants used for dyeing was performed from July 2010 to November 2011 in Congo for to inventory them and to achieve a phytochemial screening on three species selected by some number of criteria.

Methodology and Results: Interviews with herbalists, artisans, dyers and holders of traditional knowledge were done. A complete literature review of the plants of the Central African region has an inventory of one hundred and fifty (150) plants used as dyers for the people of the region. Three of these plants, Grewia coriacea Mast (Malvaceae - Grewioideae), Harungana madagascariensis Lam ex Poir. (Hypericaceae), Annickia chlorantha Oliv. (Annonaceae), were selected in terms of their frequency of use for a phytochemical screening to assess their dyeing properties. 
Conclusion and application: An ethnobotanical survey permitted to give account that the dye plants are used extensively by the population in dye and in traditional medicine. The phytochemical analysis of the three species selected omen that those can be here raw materials for the production of the dyes.

Key words: Ethnobotanical survey, dye plants, Grewia coriacea, Harungana madagascariensis, Annickia chlorantha, phytochemical screening.

\section{INTRODUCTION}

L'utilisation des colorants issus des plantes tinctoriales s'est développée au Moyen-âge, et plus tard, l'art de la teinture est devenu une industrie et la culture des plantes tinctoriales a atteint ainsi son apogée au XVIIIème siècle. Ces plantes ont permis l'obtention des colorants ayant servi à teindre les célèbres tapisseries telles que les manufactures des Gobelins et les tapisseries d'Aubuson (Cardon, 1999). Le XIXème siècle connaît l'expansion de la chimie et le développement de la chimie de synthèse qui favorisent dès le début du XXème siècle la production industrielle de l'Indigo de synthèse, et voit l'affaiblissement considérable du règne du Pastel rendant ainsi l'usage des plantes tinctoriales désuet (Graebe et al, 1869, Garfield, 2000). La mise en œuvre des ressources chromatiques constitue une alternative réaliste face aux problématiques environnementales. C'est ainsi que, l'utilisation des colorants d'origine végétale s'inscrit dans une démarche de développement durable car ils sont non toxiques, non cancérigène et biodégradables. Un sujet d'une telle envergure met le cap sur un retour vers le naturel et s'impose comme un choix dans le développement de notre région. II est donc important pour les scientifiques de soumettre à une investigation chimique les plantes utilisées traditionnellement par les populations en vue de leur valorisation. Les colorants naturels ne sont pas seulement utilisés pour donner la couleur à une variété infinie de matériaux (tissu, papier, bois, aliment,...) mais sont aussi utilisés comme substances thérapeutiques. Certains principes colorants végétaux sont pourvus de nombreuses activités pharmacologiques. C'est l'exemple du Safran d'Inde qui est une plante tinctoriale dont on extrait un colorant jaune et dont le principe tinctorial est la curcumine, le pigment majoritaire $(50-60 \%)$ responsable de cette coloration. Cette espèce chimique comme tout curcuminoïde possède une activité anticancéreuse, antioxydante, anti - inflammatoire, antibactérienne et hepatoprotectrice. Le lycopène, le pigment rouge de la tomate (Lycopersicum esculentum), est de plus en plus utilisé dans les produits de soins cutanés (Epifano et al, 2007).

En Afrique Centrale le domaine des colorants naturels n'a pas été suffisamment exploré. Le domaine reste encore non valorisé, malgré l'utilisation traditionnelle de nombreuses plantes pour leurs propriétés colorantes : Curcuma longa pour la cuisine, Lawsonia inermis (Henné) ou Pterocarpus soyauxii pour la cosmétique, Indigofera tinctoria pour les tissus, Annickia chlorantha pour la coloration des nattes (Nyegue, 2011). Peu de travaux sont disponibles dans la littérature sur les propriétés colorantes de plantes tinctoriales issues de la flore d'Afrique Centrale. Nous avons donc procédé à l'inventaire de plantes à usage tinctorial d'une part et, d'autre part réalisé un screening phytochimique des plantes sélectionnées: Grewia coriacea Mast (Malvaceae), Harungana madagascariensis Lam ex Poir (hypericaceae) et Annickia chlorantha Oliv. (Annonaceae), pour les remarquables qualités tinctoriales qu'elles présentent pour les populations, pour leur fréquence d'utilisation, leur répartition géographique à l'échelle régionale et pour la facilité d'approvisionnement. Les informations reçues des herboristes et des artisans teinturiers et les données de la littérature indiquent que l'écorce jaune soufre de tronc de Annickia chlorantha fournit un excellent colorant utilisé dans la teinture des tissus, des peaux et nattes dans certaines régions, plus particulièrement au Cameroun, au Congo, au Gabon et en RDC (Walker et al, 1995). L'extrait rouge sang des fruits de Grewia coriacea est souvent associé avec de l'argile (Kaolin) pour décorer en rouge de nombreuse parures au cours des cérémonies traditionnelles de mariage (pour le maquillage de la mariée).L'écorce de tronc de Harungana madagascariensis fournit une gomme jaune qui est utilisé comme colorant. En Ouganda, Harungana madagascariensis est une source potentielle pour 
le colorant textile (Wanyama et al, 2011). Au Cameroun, le colorant obtenu à partir de la

\section{Méthodologie}

Enquêtes ethnobotaniques : Le but de l'enquête est de rassembler autant d'information que possible au sujet des usages tinctoriaux et techniques de l'extraction des colorants végétaux. Une enquête ethnobotanique prospective a été réalisée au Congo de juillet 2010 à novembre 2011 auprès des herboristes, artisans teinturiers et détenteurs de savoir traditionnel, et compléter par une revue bibliographique sur les décoction de l'écorce sert à teindre les tissus et les nattes.

plantes de la région Afrique centrale. Trente-six personnes choisies sans tenir compte du sexe et de l'âge ont été interviewées. Un questionnaire ouvert leur a été soumis (annexe 1). A l'issue de l'enquête ethnobotanique compléter par une revue bibliographique, une liste des plantes tinctoriales a été dressée (Annexe 2, tableau 2).

\section{Annexe 1 : Fiche d'enquête}

Lieu de l'enquête :

Personne interrogée :

Artisan teinturier $\square \quad$ Détenteur de savoir traditionnel $\square \quad$ Herboristes $\square$ Autre

1- quelles sont les plantes que vous consommez ou vous connaissez et qui sont utilisées comme colorant?

2- Quelles sont les parties utilisées ?

Feuille $\square$ Fleur $\square$ écore $\square$ tige $\square$ racine $\square$ graine $\square$ Autre

3- Quel est leur type de croissance?

Arbre $\square$ arbuste $\square$ liane

herbe $\square$ Autre

4- Où les trouvez-vous ?

Forêt $\square$ savane $\square$ Autre

5- Quel est leur état

Sauvage $\square$ cultivé $\square \quad$ les deux

6- Quel est leur domaine d'application
Cosmétique
textile $\square$ alimentaire
poterie
vannerie
Autre

7- Comment préparez-vous la teinture ? Macération $\square$ décoction $\square \quad$ Autre

8- Trouvez-vous facilement ces plantes ou devez-vous aller de plus en plus loin en forêt ?

Oui $\square$ non

Récolte et identification des plantes : Les plantes récoltées dans leur milieu naturel de vie en présence d'un herboriste ou d'un détenteur de savoir sont ensuite identifiées par un botaniste. Un échantillon est déposé à l'herbarium du Centre d'études sur les
Ressources Végétales (CERVE) de Brazzaville. Les fruits de Grewia coriacea ont été récoltés dans une forêt du village Mbandaka (Imvouba) à $137 \mathrm{Km}$ sur la nationale 2 (au Nord de Brazzaville). Les écorces du tronc de Harungana madagascariensis et de Annickia 
chlorantha ont été récoltées à Owando (au Nord du Congo, département de la Cuvette).

Préparation des extraits colorants: La matière végétale est préalablement séchée et broyée. Une quantité de matière végétale a été soumise à l'extraction classique par macération. $25 \mathrm{~g}$ de poudre d'écorces ( $15 \mathrm{~g}$ de fruits) ont été macérés pendant $2 \mathrm{~h}$ à $60^{\circ} \mathrm{C}$ sous agitation mécanique dans $100 \mathrm{ml}$ d'un mélange eau/éthanol $(1: 1 \mathrm{v} / \mathrm{v})$. Après filtration et évaporation sous pression réduite, 'extrait est séché à l'étuve à $50^{\circ} \mathrm{C}$ puis broyé à l'aide d'une moulinette pour obtenir une poudre colorante.

\section{RÉSULTATS ET DISCUSSION}

Enquête ethnobotanique: 62 plantes avec leur recette de préparation ont été citées par les herboristes, artisans teinturiers et détenteurs de savoir. 34 d'entre elles ont déjà été citées comme plantes tinctoriales dans les études antérieures réalisées par Jansen et al (2005). Les 62 espèces se répartissent en 37 familles. En combinant les résultats de l'enquête sur le terrain avec ceux de la recherche bibliographique (Walker et al, 1995, Bouquet, 1969a, Bouquet, 1969b, Jansen et al, 2005, Adjanohoum et al, 1988, Adjanohoum et al, 1984, Adjanohoum et al.1981, Letouzey, 1969, Vivien et al,1985), 150 espèces tinctoriales ont été inventoriées, repartie dans 128 genres et 57 familles botaniques, dont $66 \%$ espèces sauvages, $11 \%$ espèces cultivées et $23 \%$ d'espèces sont à la fois sauvages et cultivées. L'enquête ethnobotanique nous a permis de découvrir une richesse culturelle et traditionnelle des plantes tinctoriales et sur les techniques de teinture en sculpture et masque à fabrication religieuse car il y'a encore des régions qui sont principalement animistes par rapport aux religions monothéistes. Dans l'ensemble, les espèces appartenant à la famille des Rubiaceae, Fabaceae-Faboïdeae, Fabaceaecesalpiniaceae, Anacardiaceae, apocynaceae, combretaceae et malvaceae ont été les plus citées. Ces familles à elles seules représentent plus de $50 \%$ d'espèces. Elles peuvent être donc les sources intéressantes des molécules tinctoriales, comme il l'a été prouvé chez quelques espèces appartenant à ces familles dans les travaux de Jansen et al.(2005) et de Walker et al (1995). II ressort de cette enquête que les parties des plantes les plus utilisées sont les écorces, les feuilles et les racines. Les fruits et les tiges sont moyennement utilisés et les fleurs sont moins utilisées. Plus de $50 \%$ d'espèces est utilisé traditionnellement en textile et en cosmétiques. Quant à la répartition des espèces dans la région le Gabon, le Congo, le Cameroun et la république démocratique du Congo sont des pays où l'on a recensé plus de $50 \%$ d'espèces tinctoriales. C'est au Tchad que l'on a
Screening phytochimique: Un screening phytochimique au moyen des tests de caractérisation par des réactions colorées des extraits est réalisé dans le but de déterminer les différents groupes chimiques. Ces tests ont porté sur la mise en évidence des flavonoïdes, des anthocyanes, des tanins, des composés anthracéniques et des alcaloïdes selon les techniques analytiques décrites dans les travaux de Bouquet (1969a). Les poudres colorants ont été solubilisé dans du méthanol puis filtrés et les filtrats ont servi pour les tests.

recensé le moins d'espèces. Les usages traditionnels de plantes tinctoriales diffèrent d'un pays à un autre et d'un groupe ethnique à un autre. Certaines plantes sont utilisées traditionnellement comme colorants à cause de leur aspect, de la teinture qu'elles laissent au toucher, par héritage ancestral, pour des besoins d'esthétiques ou pour des raisons culturelles et d'efficacité intrinsèque. Elles ont été utilisées traditionnellement comme source de colorants dans la teinture des cordes, du textile, de la céramique et autre parures traditionnelles. Aussi, les investigations menées ont permis de comprendre et de nous rendre compte que les populations rurales en grande majorité utilisent les mêmes plantes pour les usages thérapeutiques. Les plantes tinctoriales ont rarement fait l'objet de travaux en Afrique centrale. Certains auteurs les ont cité lors des enquêtes ethnobotaniques pour d'autres usages, surtout en médecine traditionnelle (Walker et al, 1995, Bouquet, 1969a, Bouquet, 1969b, Jansen et al, 2005, Adjanohoum et al, 1988, Adjanohoum et al, 1984, Adjanohoum et al.1981, Letouzey, 1969, Vivien et al,1985).

Phytochimie des extraits des plantes sélectionnées: Les résultats du screening phytochimique sont consignés dans le tableau 1 . L'analyse phytochimique de l'extrait de Grewia coriacea révèle une présence très remarquable des anthocyanes, la présence des flavonoïdes et des tanins condensés. Aucune étude approfondie d'un point de vue phytochimique, jusqu'à ce jour n'a été menée sur Grewia coriacea. La phytochimie semble muette sur cette espèce présumée tinctoriale nonobstant le screening chimique réalisé par Ongoka et al (2006) qui ont mis en évidence les flavonoïdes et les anthocyanes dans l'extrait aqueux. L'extrait de Harungana madagascariensis met en évidence des flavonoïdes, des tanins et les composés anthracéniques. Ces résultats corroborent avec les études phytochimiques menées sur les espèces du Cameroun, de la République démocratique du Congo, du Nigéria et d'Australie. Sur ces espèces, il a été mis en évidence 
et isolé les flavonoïdes, les tanins et les composés anthracéniques (Thin et al, 2006, Tona et al, 1998, Kouam et al, 2005, Kouam et al, 2006, Stout et al, 1962, Buckley et al, 1972, Debray et al, 1971, Ritchie et al, 1964a, Ritchie et al, 1964b, linuma et al, 1995). L'extraitde Annickia Chlorantha révèle la présence des alcaloïdes et des flavonoïdes. Les travaux de Dongmo et al (2007) sur Annickia chlorantha ont mis en évidence ces familles chimiques. D'après les travaux de Harmonniere et al (1975), Samir et al (1989)et Oliver-Bever (1983)plusieurs alcaloïdes quaternaires ont été isolés chez l'espèce Annickia Chlorantha. Cardon (2003) signale que ces alcaloïdes quaternaires souvent présents dans les plantes sous forme de sels (chlorure, sulfates principalement) pouvaient être des principes tinctoriaux des colorants jaunes basiques. II s'agit de la berbérine, de la jatrorrhizine, de la columbamine et de la palmatine. De nombreuses études attribuent aux flavonoïdes, anthocyanes, tanins, composés et dérivés anthracéniques et alcaloïdes quaternaires des propriétés tinctoriales (Cardon, 2003, Brouillard, 1993, Brouillard et al, 1977, Clifford, 2000, Wilska-Jeszka, 1991). La présence de ces familles chimiques dans les espèces choisie pour notre étude, peuvent être mis à profit pour les proposer comme des sources potentielles de colorants naturels. Leur utilisation traditionnelle comme colorants est donc bien fondée.

Tableau 1.: Screening chimique des extraits de Grewia coriacea, Harungana madagascariensis et Enantia chlorantha par réactions colorées.

\begin{tabular}{|l|c|c|c|}
\hline Groupes chimiques & Grewia coriacea & Harungana madagascariensis & Annickia chlorantha \\
\hline Flavonoïdes & + & + & + \\
Anthocyanes & ++ & - & - \\
anthraquinones & - & ++ & - \\
Alcaloïdes & - & - & ++ \\
Tanins & + & + & \pm \\
\hline
\end{tabular}

++ : Présence très remarquable ; + : présence ; \pm : présence peu remarquable ; - : absent.

\section{CONCLUSION}

Nous avons entrepris un inventaire ethnobotanique et menée une évaluation des propriétés tinctoriales de trois plantes utilisées en Afrique centrale comme colorant, à partir du screening chimique. Les flavonoïdes, les anthocyanes, les anthraquinones, les quinones et les alcaloïdes (substances naturelles à intérêt tinctorial considérable) mis en évidence par les tests phytochimiques pourraient présager que Grewia coriacea, Harungana madagascariensis et Annickia chlorantha sont doués de propriété tinctoriale. Ces résultats restent préliminaires, il serait intéressant d'isoler les molécules qui sous-tendent les propriétés tinctoriales. De plus, des études complémentaires approfondies concernant l'identification de ces

\section{Remerciement}

Nous remercions sincèrement l'Unité de Chimie de la vie et du végétal (UC2V) pour nous avoir permis de

\section{RÉFÉRENCES BIBLIOGRAPHIQUES}

Adjanohoum, Ahyia MR, Aké-Assi L, et al, [1988]. Contribution aux études ethnobotaniques et floristiques en république du Congo. Paris, Edition ACCT, p 605.

Adjanohoum, E.J. et al. [1984]. Contribution aux études ethnobotaniques et floristiques au Gabon. Ed. ACCT. 294p. composés par des méthodes plus performantes sont nécessaires. Pour tirer profit de l'information brute qui inonde le territoire concernant ces plantes utilisées traditionnellement par héritage ancestral comme colorant, à cause de leur texture, ou pour les besoins esthétiques, il a été nécessaire d'établir un listing de plantes. En posant donc la fondation d'une banque de données, nous offrons l'accès direct à l'information basique et essentielle souhaitée c'est-à-dire une information de référence disponible pour de nombreux utilisateurs. Ce travail de base, vise à contribuer à une plus grande connaissance des plantes tinctoriales d'Afrique centrale ainsi qu'à leur valorisation comme sources de colorants ou de teintures naturelles.

réaliser les enquêtes sur le terrain par son soutien financier.

Adjanohoum, E.J. et al.[1981]. Contribution aux études ethnobotaniques et floristiques en République centrafricaine. Ed. ACCT. 139p.

Bouquet A. [1969a] - Féticheur et médecine traditionnelle du Congo. Mémoire ORSTOM $n^{\circ} 36$, Paris - France, $282 \mathrm{P}$. 
Bouquet, A.[1969b]. Féticheurs et médecines traditionnelles du Congo (Brazzaville). Ed. ORSTOM. 282p.

Brouillard R. [1993] - The Flavonoids, Advances in research since 1986, éd. J.B.Harborne, Chapman and Hall, London, 525-538.

Brouillard, R. and B. Delaporte [1977]. "Chemistry of anthocyanin pigments. II kinetic and thermodynamic study of proton transfer, hydration and tautomeric reactions of malvidin- 3-glucoside." J. Am. Chem.Soc. 99: 8461-8468.

Buckley, D.G., Ritchie, E., Taylor, W.C., Young, L.M. [1972]. Aust. J. Chem. 25, 843

Cardon, D. [1999]- Les teintures naturelles. Pour la science, 266, 50-55.

Cardon, D. [2003]-Le monde des teintures naturelles, Edition Belin, ISBN 2-7011-2678-9, 586p.

Clifford, M. N. [2000]. "Anthocyanins-nature, occurrence and dietary burden- a review." $J$. Sci. Food Agri. 80: 1063-1072. Colorants, Blackie Acadenamic ans professional: 244280.

Debray, M., Jacquemin, H.,Razafindrambao, R. [1971] - Contribution à l'inventatire des plantes médicinales de Madagascar. Travaux et Documents de l'ORSTOM, N 8,1 vol. 150 $\mathrm{pp}$.

Dongmo, G.I et al. [2007]. Phytochemical constituents and antioxidant potential of some Cameroonian medicinal plants, Pharmacology online 2: 436-452.

Epifano, F., Genovese, S., Menghini, L., Curini, M. [2007]. Chemistry and pharmacology of oxyprenylated secondary plant metabolites. Phytochemistry, 68: 939-953.

Garfield, Simon Mauve [2000] - How One Man Invented a Color that Changed the World.

Graebe, C et Liebermann, C. [1869]. Ueberanthracen und alizarin. Annalen der chemie und pharmacie supplement - band, , 7, 257-322.

Harmonniere, M., Lebœuf, M., Cave, A et Paris, R. [1975]. Pl. Méd. Phytoch. 9, P. 296.

linuma, M., Tosa, H., Ito, T., Tanaka, T., Aqil, M. [1995]. Phytochemistry 40, 267.

Jansen, P.C.M et Cardon, D. [2005]. Ressources végétales de l'Afrique tropicale 3 . Colorants et tanins. Fondation PROTA, CTA, Wageningen, Pays- Bas. 238 pp.

Kouam, F. S., Ngadjui T., \&coll.[2005]. Prenylatedanthronoild antioxidants from the stem bark of Harungana madagascariensis. Phytochemistry 66: 11741179 pp.
Kouam, F. S., Shamsun Nahar Khan \&coll. [2006]. $\alpha-$ Glucosidase Inhibitory Anthranols, Kenganthranol A - C, from the stem Bark of Harungana madacascariensis. J. Nat. Prod. 69: $229-233 \mathrm{pp}$.

Letouzey, R.[1969]. Manuel de botanique tropicale. C.T.F.T. Tome 2a, $p 11$

Nyegue, M. A. [2011]- Dye plants of Cameroon, from tradition to new research. International Symposium and Exhibition on Natural Dyes, La Rochelle, France 24 - 30 April.

Oliver-Berver, B. [1983] - Medicinal plants in tropical west Africa II. Plants acting on the nervous system. Journal of Ethnopharmacology, 7,193.

Ongoka P. R., Matini L., Moutou J. M., YouhouvoulouNgabé D. [2006]. Évaluation des propriétés physico-chimiques et du profil chimique des indicateurs colorés naturels locaux. Cas des extraits de : hibiscus sabdariffa, amaranthus oleraceus, Grewia coriacea, spinacia oleracea et bougainvillea spectabilis. Annales de l'Université Marien NGOUABI, 7 (3) : 138-146 pp.

Profizi, J.P., Makita-Madzou, J.P., Milandou, J.C., Karanda, C.N., Motom, M. \&Bitsindou, I. [1993]. Ressources végétales non-ligneuses des forêts du Congo. Rapport du programme d'action forestier du Congo. Brazzaville

Ritchie, E., Taylor, W.C. [1964a]. Tetrahedron Lett. 23, 1431.

Ritchie, E., Taylor, W.C., Shannon, J.S. [1964b]. Tetrahedron Lett. 23, 1437

Samir, A. R., Makboul, A. M., Moses, O. F., Gabriel O., Zac, G. [1989], Bull. Fac. Sci, 18 (2), 21-9.

Stout, G.H., Alden, R.A., Kraut, J., Taylor, W.C., Young, L.M., [1962]. J. Am. Chem.Soc. 84, 1653.

Thin A. E., Ghogomu R.T., Sondengam B.L \& coll. [2006]. Minor constituents of Harungana madacascariensis stembark. Biochimie systematics and ecology 34: $267-269$ pp.

Tona, L., Kambu, K., Ngimbi, N., Cimanga, K. and Vlietinck, A.J. [1998]. Antiamoebic and phytochemical screening of some Congolese medicinal plants. Journal of Ethnopharmacology61: 57-65.

Vivien, J., Faure, I. J. [1985]. Arbres des forêts denses d'Afrique Centrale. Paris, France : ACCT.

Walker, A. R et Sillans, R. [1995]- Les plantes utiles du Gabon. Edition Sépia, ISBN 2-907888-69-2, 614p.

Wanyama, P.A.G., Kiremire, B.T., Ogwok, P. and Murumu, J.S. [2011]. Indigenous plants in Uganda as potential sources of textile dyes. 
African Journal of Plant science. Vol. 5 (1), pp. 28-39.
Wilska-Jeszka, J. [1991]. "Anthocyanins as natural food colorants." IFI 3: 10-15. 


\section{Annexe 2 : Résultats de l'inventaire des plantes tinctoriales d'Afrique centrale}

* Espèces citées dans les enquêtes, ${ }^{* *}$ Espèces citées dans les enquêtes et dans la bibliographie.

Tableau 2: Résultats de l'inventaire des plantes tinctoriales d'Afrique centrale

\begin{tabular}{|c|c|c|c|c|c|c|c|c|c|}
\hline Famille & Genre-espèce & Nom commun & État & $\begin{array}{l}\text { Partie } \\
\text { utilisée }\end{array}$ & $\begin{array}{l}\text { Type } \\
\text { croiss. }\end{array}$ & Répartition & Principes tinctoriaux & $\begin{array}{l}\text { Couleur de } \\
\text { l'extrait }\end{array}$ & $\begin{array}{l}\text { Usages } \\
\text { tinctoriaux }\end{array}$ \\
\hline Amaranthaceae & Amaranthus spinosus L. * & Amarante rouge & Sauv. & $\mathrm{F}$ & Herbe & $\begin{array}{l}\mathrm{CG}, \mathrm{GE}, \mathrm{GB}, \mathrm{CM} \text {, } \\
\mathrm{CD}, \mathrm{RW}\end{array}$ & anthocyanes & rouge & Alimentaire \\
\hline Alliaceae & Allium sepa L. & Oignon & Cult. & Pelure & Herbe & Toute la région & $\begin{array}{l}\text { Flavonols, tannins, } \\
\text { anthocyanes }\end{array}$ & Rouge-violet & Alimentaire \\
\hline Acanthaceae & $\begin{array}{l}\text { Whitfieldia longifolia T. } \\
\text { And }\end{array}$ & - & Sauv. & $F$ & Arbuste & $\mathrm{GB}, \mathrm{CG}$ & - & Noire & Non rapporté \\
\hline \multirow{5}{*}{ Anacardiaceae } & $\begin{array}{l}\text { Lannea barteri (Oliv.) } \\
\text { Eng|** }\end{array}$ & - & Sauv. & $E$ & Arbre & $\mathrm{CD}, \mathrm{BU}$ & - & $\begin{array}{l}\text { Brun-rouge- } \\
\text { orangé }\end{array}$ & Textile \\
\hline & $\begin{array}{l}\text { Lannea microcarpa Engl. } \\
\text { et Kn }\end{array}$ & Vrai raisinier & Sauv. & $E$ & Arbre & $\mathrm{CM}$ & Anthocyanines & Rouge -brun & Textile \\
\hline & Lannea velutina A. Rich & Raisinier velu & Sauv. & $E$ & Arbre & GU, Td, CA. & - & Brun-rouge & textile \\
\hline & Spondias mombin L. ${ }^{*}$ & $\begin{array}{l}\text { Prune } \\
\text { mombin }\end{array}$ & Sauv. & $\mathrm{Fr}$ & Arbre & $\mathrm{CG}, \mathrm{GB}, \mathrm{CD}$ & - & jaune & Alimentaire \\
\hline & Mangifera indica L. ${ }^{*}$ & Manguier & Sauv\&cult. & $F, E$ & Arbre & Toute la région & - & Jaune-brun & Non indiqué \\
\hline \multirow{4}{*}{ Annonaceae } & $\begin{array}{l}\text { Xylopia eathiopica A. } \\
\text { Rich. }{ }^{*}\end{array}$ & - & Sauv. & $\mathrm{R}$ & $\begin{array}{l}\text { Arbre / } \\
\text { arbuste }\end{array}$ & $\begin{array}{l}\text { CG, GU, GB } \\
\mathrm{CA}\end{array}$ & - & jaune & Vannerie \\
\hline & Annona senegalensis & & Sauv. & $E$ & arbuste & & - & Rose marron & \\
\hline & Annona reticulata L. & Cœur de boeuf & Sauv. & $\mathrm{Fr}$ & $\begin{array}{l}\text { Arbre/ } \\
\text { arbuste }\end{array}$ & CG, GB & - & Pourpre & Non rapporté \\
\hline & $\begin{array}{l}\text { Annickia chlorantha Oliv. } \\
* *\end{array}$ & $\begin{array}{l}\text { Bois jaune } \\
\text { africain }\end{array}$ & Sauv. & $E$ & Arbre & Toute la région & $\begin{array}{l}\text { Alcaloïdes } \\
\text { quaternaires }\end{array}$ & Jaune & Non indiqué \\
\hline \multirow{3}{*}{ Apocynaceae } & Landophia $s p^{*}$ & - & Sauv. & $\mathrm{Fr}$ & Liane & CG, GE, GB & - & jaune & Alimentaire \\
\hline & $\begin{array}{l}\text { Clitandra cymolosa } \\
\text { Benth.* }^{*}\end{array}$ & - & Sauv. & $\mathrm{Fr}$ & Liane & CG & - & $\begin{array}{l}\text { Rouge- } \\
\text { orangé }\end{array}$ & Non indiqué \\
\hline & $\begin{array}{l}\text { Saba comorensis (Bojer } \\
\text { ex DC.) Pichon * }\end{array}$ & Liane goïne & Sauv. & $\mathrm{Fr}$ & Liane & $\begin{array}{l}\text { CG, GB, CM, CA, } \\
\text { CD }\end{array}$ & $\begin{array}{l}\text { Carotenoïdes, } \\
\text { anthraquinones }\end{array}$ & jaune & Non indiqué \\
\hline Asphodelaceae & Aloe zebrina Baker & $\begin{array}{l}\text { Aloès zébré, } \\
\text { aloès tacheté }\end{array}$ & Sauv. & $\mathrm{R}, \mathrm{F}$ & Herbe & CD & $\begin{array}{l}\text { C-hétérosides } \\
\text { d'anthrones }\end{array}$ & Jaune d'or & vannerie \\
\hline Phyllanthaceae & $\begin{array}{l}\text { Bridelia ferruginea Benth. } \\
* *\end{array}$ & Bridelia & Sauv. & $E$ & Arbuste & Toute la région & & Rouge & Non indiqué \\
\hline
\end{tabular}

$\mathrm{F}=$ feuille, $\mathrm{fl}=$ fleure, $\mathrm{Fr}=$ fruit, $\mathrm{E}=$ écorce, $\mathrm{Er}=$ écorce racine, $\mathrm{R}=$ racine, $\mathrm{G}=$ graine, $\mathrm{T}=$ tige, $\mathrm{Rmf}=$ rameaux feuillée, $\mathrm{Lt}=$ latex, $\mathrm{Tb}=\mathrm{tubercule}, \mathrm{Rz}=$ rhizome, $\mathrm{B}=$ bois, $\mathrm{Pl}=$ plante CM: Cameroun, CG : Congo, ST : Sao Tomé et principe, Td : Tchad, CA : Centrafrique, CD : Congo Démocratique, GE : Guinée Equatoriale, BU : Burundi, RW : Rwanda, GB : Gabon 
Tableau 2(suite 1) : Résultats de l'inventaire des plantes tinctoriales d'Afrique centrale

\begin{tabular}{|c|c|c|c|c|c|c|c|c|c|}
\hline Famille & Genre-espèce & $\begin{array}{l}\text { Nom } \\
\text { commun }\end{array}$ & État & $\begin{array}{l}\text { Partie } \\
\text { utilisée }\end{array}$ & $\begin{array}{l}\text { Type } \\
\text { croiss. }\end{array}$ & Répartition & $\begin{array}{l}\text { Principes } \\
\text { tinctoriaux }\end{array}$ & $\begin{array}{l}\text { Couleur de } \\
\text { l'extrait }\end{array}$ & $\begin{array}{l}\text { Usages } \\
\text { tinctoriaux }\end{array}$ \\
\hline \multirow{3}{*}{ Astéraceae } & Cosmos sulphureus Cav. & $\begin{array}{l}\text { Cosmos } \\
\text { soufré }\end{array}$ & Cult. & $\mathrm{FI}$ & herbe & CM. & $\begin{array}{l}\text { Composés } \\
\text { flavonoïdes }\end{array}$ & jaune & textile \\
\hline & Tagetes erecta L. & Rose d'inde & Cult. & $\mathrm{FI}$ & Herbe & Toute la région & $\begin{array}{l}\text { Hétérosides de } \\
\text { flavonoïdes }\end{array}$ & jaune & Alimentaire, textile \\
\hline & Bidens pilosa L. * & & Sauv. & $\mathrm{T}, \mathrm{F}$ & Herbe & Toute la région & & $\begin{array}{l}\text { Jaune } \\
\text { rougeâtre }\end{array}$ & Non indiqué \\
\hline Bombacaceae & $\begin{array}{l}\text { Ceiba pentadra (Linn.) } \\
\text { Gaertn }\end{array}$ & Fromager & Sauv. & $E$ & Arbre & Toute la région & $\begin{array}{l}\text { Quercetol et } \\
\text { kaempferol }\end{array}$ & Noirâtre & \\
\hline Brassicaceae & Brassica oleracea & Chou rouge & Cult. & $F$ & Legume & $\begin{array}{l}\text { Afrique } \\
\text { centrale }\end{array}$ & anthocyanes & Bleu, violet & alimentaire \\
\hline Balsaminaceae & Impatiens tinctoria A.Rich. & Balsamine & Sauv. & $\mathrm{R}, \mathrm{Tb}$ & Herbe & $\mathrm{CD}, \mathrm{RW}, \mathrm{BU}$ & polyphénols & $\begin{array}{l}\text { Rouge } \\
\text { sombre }\end{array}$ & Cosmétique \\
\hline Bassellaceae & Basella nigra Lour. ** & $\begin{array}{l}\text { Epinard des } \\
\text { indes }\end{array}$ & Cult. & $\mathrm{F}$ & Herbe & $\begin{array}{l}\text { CG,CD, GB, } \\
\text { RW, BU }\end{array}$ & - & Rouge & $\begin{array}{l}\text { Teinture, } \\
\text { cosmétique }\end{array}$ \\
\hline \multirow[t]{2}{*}{ Bignoniaceae } & $\begin{array}{l}\text { Arrabidée chica (Bompl.) B. } \\
\text { verl }\end{array}$ & $\begin{array}{l}\text { Cricket - } \\
\text { vine }\end{array}$ & Sauv. & - & Herbe & CM, ST, GB & anthocyanes & $\begin{array}{l}\text { Rouge / } \\
\text { pourpre }\end{array}$ & cosmétique \\
\hline & $\begin{array}{l}\text { Stereospermum kunthianum } \\
\text { Cham. }\end{array}$ & & Sauv. & $E$ & Arbre & Toute la région & - & Rouge-brun & Cosmétique \\
\hline Bixaceae & Bixa orellana L. ** & $\begin{array}{l}\text { Rocouyer, } \\
\text { roucou }\end{array}$ & $\begin{array}{l}\text { Sauv. } \\
\text { \&cult. }\end{array}$ & $G$ & $\begin{array}{l}\text { Arbre / } \\
\text { arbuste }\end{array}$ & Toute la région & caroténoïdes & $\begin{array}{l}\text { Rouge / } \\
\text { jaune-orangé }\end{array}$ & $\begin{array}{l}\text { Alimentaire } \\
\text { cosmétique, textile }\end{array}$ \\
\hline Boraginaceae & $\begin{array}{l}\text { Arnebia hispidissima } \\
\text { (Sieber ex Lehm.) DC. }\end{array}$ & $\begin{array}{l}\text { Sang de } \\
\text { l'homme }\end{array}$ & $\begin{array}{l}\text { Sauv. } \\
\text { \&cult }\end{array}$ & $\mathrm{R}$ & Herbe & $\mathrm{CM}, \mathrm{Td}$. & naphtoquinone & Rouge violacé & alimentaire, textile \\
\hline \multirow{3}{*}{ Burseraceae } & Santira trimeria (oliv) Aubr. & - & Sauv. & $E$ & Arbre & CG, GE & - & rougeâtre & Non indiqué \\
\hline & Pachylobus edulis G. Don. & - & Cult. & $\mathrm{F}$ & Arbre & CG, GB & - & Noire & Non indiqué \\
\hline & Dacryodes macrophylla** & - & Sauv. & $\mathrm{F}$ & Arbuste & $\mathrm{GB}, \mathrm{CG}, \mathrm{GE}$ & - & Rouge violacé & Non indiqué \\
\hline Chenopodiaceae & Spinacia oleracea* & Epinard & Cult. & $G$ & herbe & Toute la région & - & Rouge violacé & Indcateur coloré \\
\hline Composeae & Eclipta Alba Hassk. & & Sauv. & $\mathrm{F}$ & Herbe & GB & - & Noir & Non rapporté \\
\hline Lamiaceae & Vitex madiensis Oliv. ${ }^{*}$ & - & Sauv. & $\mathrm{Fr}$ & Arbuste & CG, GE & - & Noire & Tatouage \\
\hline Apiaceae & Daucus Carota L. ** & Carotte & Cult. & $\mathrm{Rz}$ & plante & Toute la région & caroténoïdes & Orange & $\begin{array}{l}\text { Alimentaire, } \\
\text { cosmétique }\end{array}$ \\
\hline
\end{tabular}

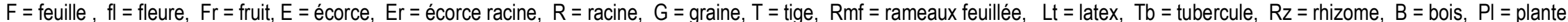
CM: Cameroun, CG : Congo, ST : Sao Tomé et principe, Td : Tchad, CA : Centrafrique, CD : Congo Démocratique, GE : Guinée Equatoriale, BU : Burundi, RW : Rwanda, GB : Gabon. 
Tableau 2 (suite 2): Résultats de l'inventaire des plantes tinctoriales d'Afrique centrale

\begin{tabular}{|c|c|c|c|c|c|c|c|c|c|}
\hline Famille & Genre-espèce & Nom commun & État & $\begin{array}{l}\text { Partie } \\
\text { utilisée }\end{array}$ & $\begin{array}{l}\text { Type } \\
\text { croiss. }\end{array}$ & Répartition & Principes tinctoriaux & $\begin{array}{l}\text { Couleur de } \\
\text { l'extrait }\end{array}$ & $\begin{array}{l}\text { Usages } \\
\text { tinctoriaux }\end{array}$ \\
\hline \multirow{12}{*}{$\begin{array}{l}\text { Fabaceae - } \\
\text { Cesalpiniaceae }\end{array}$} & $\begin{array}{l}\text { Caesalpinia coriaria (Jacq.) } \\
\text { Willd**}\end{array}$ & Dividivi & Cult. & $\mathrm{B}, \mathrm{Fr}$ & Arbre & $C D$ & Composés aucubines & $\begin{array}{l}\text { Noire, bleue } \\
\text {,rouge }\end{array}$ & $\begin{array}{l}\text { textile et } \\
\text { poterie }\end{array}$ \\
\hline & Caesalpinia sapan L. ${ }^{* *}$ & $\begin{array}{l}\text { Sapan, bois } \\
\text { de sapan }\end{array}$ & Cult. & $\mathrm{B}, \mathrm{Fr}$ & Arbre & $C D$ & $\begin{array}{l}\text { Braziline, composés } \\
\text { phénoliques }\end{array}$ & $\begin{array}{l}\text { rouge (bois), } \\
\text { noire (fruit) }\end{array}$ & $\begin{array}{l}\text { alimentaire, } \\
\text { textiles. }\end{array}$ \\
\hline & Bandeira eatenuiflora Benth. & & Sauv. & $\mathrm{F}$ & Liane & GB & - & Noire & Non indiqué \\
\hline & Copaifora Le testui Pell. & & Sauv. & $E$ & Arbre & GB & - & Noire & Textile \\
\hline & Eurypetalum batesii Bak. F. & & Sauv. & $\mathrm{B}$ & Arbre & GB & - & Rouge & Non indiqué \\
\hline & GriffoniaphysocarpaBaill & - & Sauv. & $\mathrm{F}, \mathrm{R}$ & $\begin{array}{l}\text { Arbuste / } \\
\text { liane }\end{array}$ & $\begin{array}{l}\text { CG, CD, CM, } \\
\mathrm{GB}, \mathrm{GE}\end{array}$ & - & Noire & cosmétique \\
\hline & $\begin{array}{l}\text { Haematoxylum campechianum } \\
\text { L. }\end{array}$ & $\begin{array}{l}\text { Arbre à } \\
\text { Campêche }\end{array}$ & Cult. & B & $\begin{array}{l}\text { Arbre I } \\
\text { arbuste }\end{array}$ & $\mathrm{CD}, \mathrm{GB}$ & Hématoxyline & Rouge & Textile, encre \\
\hline & $\begin{array}{l}\text { Piliostigma thonningü (Schm } \\
\text { Milne- Redhead) }\end{array}$ & & Sauv. & $\mathrm{E}$ & Arbre & Toute la région & - & Rouge - brun & Non indiqué \\
\hline & $\begin{array}{l}\text { Pterolobium stellatum (Forssk.) } \\
\text { Brenan ** }\end{array}$ & $\begin{array}{l}\text { Mutanda } \\
\text { (swahili) }\end{array}$ & $\begin{array}{l}\text { Sauv. } \\
\text { \&cult. }\end{array}$ & $E, F$ & Arbuste & Toute la région & - & Rouge vif & Textile, encre \\
\hline & Senna auriculata (L.) Roxb & Avaram & $\begin{array}{l}\text { Sauv. } \\
\text { \&cult }\end{array}$ & $\mathrm{E}, \mathrm{Fr}, \mathrm{G}$ & $\begin{array}{l}\text { Arbre / } \\
\text { arbuste }\end{array}$ & $C A, C D$ & anthraquinones & $\begin{array}{l}\text { Jaune, rouge } \\
\text { noir }\end{array}$ & Textile \\
\hline & Cassia alata* & Dartrier & & $\mathrm{R}$ (jus) & Arbuste & Toute la région & $\begin{array}{l}\text { Anthraquinones, } \\
\text { flavanol }\end{array}$ & Noire & Tatouage \\
\hline & Tamarindus indica L. & Tamarinier & Sauv. & $E$ & arbuste & $\mathrm{CD}, \mathrm{CA}, \mathrm{CM}$ & - & $\begin{array}{l}\text { Brun-rouge, } \\
\text { beige }\end{array}$ & Non indiqué \\
\hline Fabaceae & $\begin{array}{l}\text { Haematoxy lumbrasiletto } \mathrm{H} \text {. } \\
\text { karst }\end{array}$ & Bois du brésil & $\begin{array}{l}\text { Sauv. } \\
\text { \&cult }\end{array}$ & $\mathrm{E}$ & Arbre & CM, ST & Braziline & Rouge sang & Textile, \\
\hline
\end{tabular}

$\mathrm{F}=$ feuille, $\mathrm{fl}=$ fleure, $\mathrm{Fr}=$ fruit, $\mathrm{E}=$ écorce, $\mathrm{Er}=$ écorce racine, $\mathrm{R}=$ racine, $\mathrm{G}=$ graine, $\mathrm{T}=$ tige, $\mathrm{Rmf}=$ rameaux feuillée, $\mathrm{Lt}=$ latex, $\mathrm{Tb}=$ tubercule, $\mathrm{Rz}=$ rhizome, $\mathrm{B}=$ bois, $\mathrm{Pl}=$ plante. CM: Cameroun, CG : Congo, ST : Sao Tomé et principe, Td : Tchad, CA : Centrafrique, CD : Congo Démocratique , GE : Guinée Equatoriale, BU : Burundi, RW : Rwanda, GB : Gabon. 
Tableau 2 (suite 3): Résultats de l'inventaire des plantes tinctoriales d'Afrique centrale

\begin{tabular}{|c|c|c|c|c|c|c|c|c|c|}
\hline Famille & Genre-espèce & Nom commun & État & $\begin{array}{l}\text { Partie } \\
\text { utilisée }\end{array}$ & $\begin{array}{l}\text { Type } \\
\text { croiss. }\end{array}$ & Répartition & $\begin{array}{l}\text { Principes } \\
\text { tinctoriaux }\end{array}$ & $\begin{array}{l}\text { Couleur de } \\
\text { l'extrait }\end{array}$ & Usages tinctoriaux \\
\hline \multirow{5}{*}{ Combretaceae } & $\begin{array}{l}\text { Anogeissus leiocarpa (DC. } \\
\text { ) Guil. \&Perr** }\end{array}$ & $\begin{array}{l}\text { bouleau } \\
\text { d'Afrique }\end{array}$ & $\begin{array}{l}\text { Sauv. } \\
\text { \&cult }\end{array}$ & $F, E, R$ & $\begin{array}{l}\text { Arbre } \\
\text { larbuste }\end{array}$ & $\begin{array}{l}\text { Td., CA, CM., } \\
\text { CD, CG }\end{array}$ & flavonoïdes & $\begin{array}{l}\text { Jaune, ocre, } \\
\text { ocre-rouge }\end{array}$ & Textile \\
\hline & $\begin{array}{l}\text { Combretum glutinosum } \\
\text { Perr ex DC. }{ }^{* *}\end{array}$ & $\begin{array}{l}\text { Bois } \\
\text { d'éléphant }\end{array}$ & Sauv. & $\mathrm{F}$ & Arbuste & $\begin{array}{l}\mathrm{GE}, \mathrm{Td}, \mathrm{CA} \\
\mathrm{CM}, \mathrm{CG}\end{array}$ & flavonoïdes & jaune brunâtre & Textile \\
\hline & $\begin{array}{l}\text { Languncularia racemosa } \\
\text { (L.) C.F.Gaertn }\end{array}$ & $\begin{array}{l}\text { Palétuvier } \\
\text { blanc }\end{array}$ & Sauv. & $E, F$ & $\begin{array}{l}\text { Arbre / } \\
\text { arbuste }\end{array}$ & GB, CG, GE. & - & Brun & Non indiqué \\
\hline & Terminalia chebula Retz. & Cadou & Cult. & $\mathrm{Fr}, \mathrm{FI}$ & arbre & $\mathrm{CD}$ & $\begin{array}{l}\text { Composés } \\
\text { phénoliques }\end{array}$ & Jaune, brun. & Ingrédient auxiliaire \\
\hline & $\begin{array}{l}\text { Terminalia superba Engl. } \\
\text { Et Diels** }^{* *}\end{array}$ & $\begin{array}{l}\text { Noyer du } \\
\text { mayombe }\end{array}$ & Sauv. & $E$ & Arbre & CG, GB & - & Noire & Textile \\
\hline \multirow{4}{*}{ Ebenaceae } & Euclea divinorum Hiern & Mdala (swahili) & Sauv. & $\mathrm{E}, \mathrm{Fr}, \mathrm{R}$ & $\begin{array}{l}\text { Arbre / } \\
\text { arbuste }\end{array}$ & $\mathrm{CD}, \mathrm{RW}, \mathrm{BU}$ & flavonoïdes & $\begin{array}{l}\text { Rouge-brun, } \\
\text { marron }\end{array}$ & Textile, vannerie \\
\hline & $\begin{array}{l}\text { Diospyros heterotricha } \mathrm{F} \text {. } \\
\text { White }^{* *}\end{array}$ & - & $\begin{array}{l}\text { Sauv. } \\
\text { \&cult. }\end{array}$ & $\mathrm{T}, \mathrm{R}$ & $\begin{array}{l}\text { Arbre / } \\
\text { arbuste }\end{array}$ & CG, CD & - & Jaune-orangé & cosmétique \\
\hline & $\underset{* *}{\text { Diospyros geletti De Wild }}$ & Ebene & Sauv. & $\mathrm{F}$ & Arbuste & Toute la région & & Rouge & Cosmétique \\
\hline & $\begin{array}{l}\text { Diospyro smespiliformis } \\
\text { Hochst. Ex A.DC * }\end{array}$ & Ebene & Sauv. & & Arbuste & $\mathrm{CM}$ & naphtoquinone & Kaki & Cosmétique \\
\hline \multirow[t]{3}{*}{ Euphorbiaceae } & $\begin{array}{l}\text { Erythrococa Chevalieri } \\
\text { (Beille ) Prain* }\end{array}$ & - & Sauv. & $\mathrm{F}$ & Arbuste & $\begin{array}{l}\mathrm{CG}, \mathrm{CD}, \mathrm{RW} \\
\mathrm{BU}, \mathrm{GB}\end{array}$ & - & Bleue & Tatouage \\
\hline & $\begin{array}{l}\text { Bridelia micrantha (Hochst) } \\
\text { Baill. * }\end{array}$ & & Sauv. & $E, F, B$ & Arbuste & Toute la région & - & Brun & $\begin{array}{l}\text { Teinture pour la } \\
\text { céramique }\end{array}$ \\
\hline & Alchorne cordifolia Mull. * & & Sauv. & $E, F$ & Arbuste & $\mathrm{GB}, \mathrm{CG}$ & - & Noir & Textile, poterie \\
\hline Vitaceae & Vitis vinifera $\mathrm{L}^{* *}$ & Vigne rouge & $\begin{array}{l}\text { Sauv. } \\
\text { \&cult }\end{array}$ & $\mathrm{Fr}$ & Arbuste & $\begin{array}{l}\text { CG, CD, CM, } \\
\text { GE }\end{array}$ & anthocyanosides & $\begin{array}{l}\text { Violette, bleue } \\
\text { et grise }\end{array}$ & Fabrication du vin \\
\hline
\end{tabular}

$\mathrm{F}=$ feuille, $\mathrm{fl}=$ fleure, $\mathrm{Fr}=$ fruit, $\mathrm{E}=$ écorce, $\mathrm{Er}=$ écorce racine, $\mathrm{R}=$ racine, $\mathrm{G}=$ graine, $\mathrm{T}=$ tige, $\mathrm{Rmf}=$ rameaux feuillée, $\mathrm{Lt}=$ latex, $\mathrm{Tb}=$ tubercule, $\mathrm{Rz}=$ rhizome, $\mathrm{B}=$ bois, $\mathrm{Pl}=$ plante CM: Cameroun, CG : Congo, ST : Sao Tomé et principe, Td : Tchad, CA : Centrafrique, CD : Congo Démocratique, GE : Guinée Equatoriale, BU : Burundi, RW : Rwanda, GB : Gabon. 
Tableau 2 (suite 4): Résultats de l'inventaire des plantes tinctoriales d'Afrique centrale

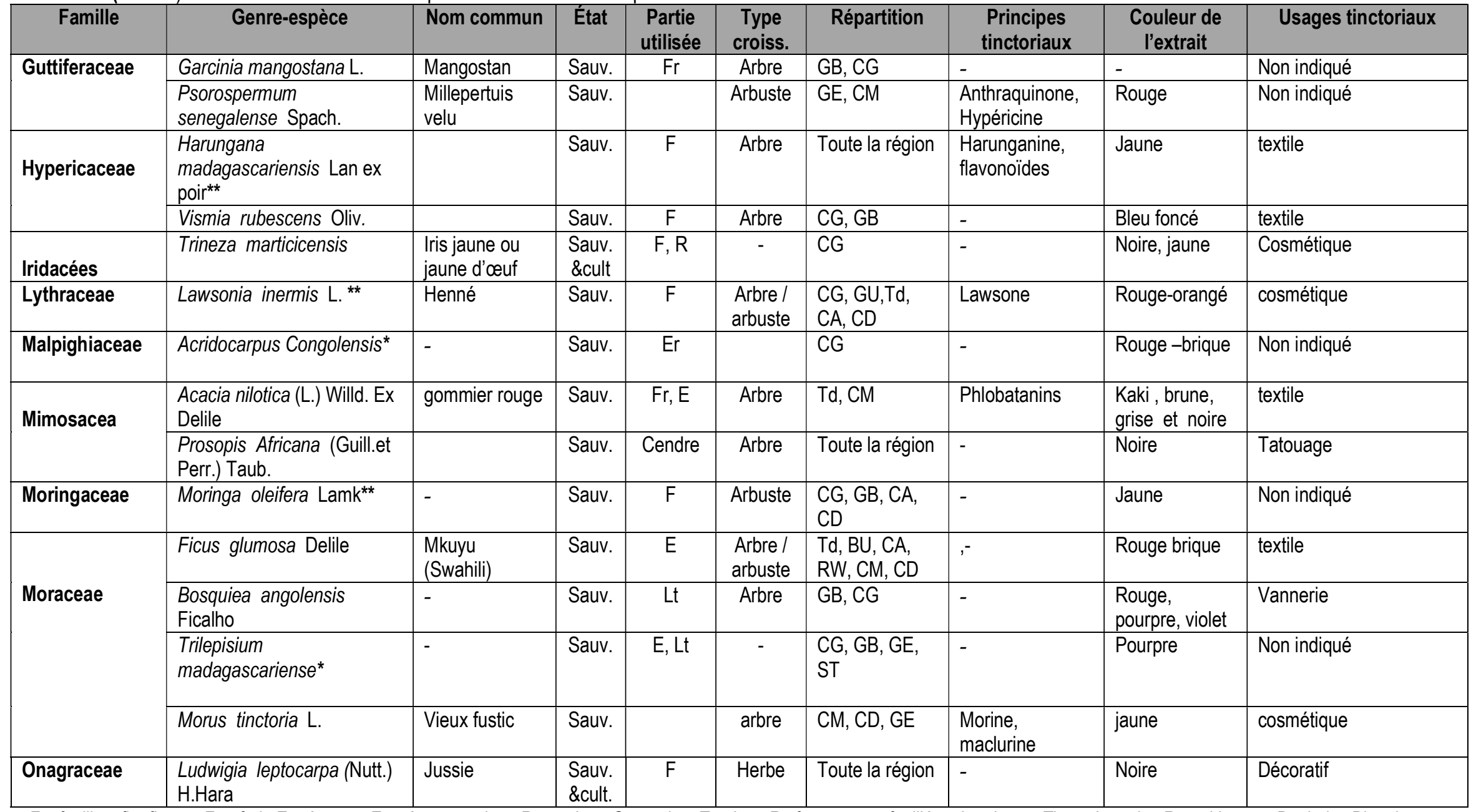

$\mathrm{F}=$ feuille, $\mathrm{fl}=$ fleure, $\mathrm{Fr}=$ fruit, $\mathrm{E}=$ écorce, $\mathrm{Er}=$ écorce racine, $\mathrm{R}=$ racine, $\mathrm{G}=$ graine $\mathrm{T}=$ tige, $\mathrm{Rmf}=$ rameaux feuillée, $\mathrm{Lt}=$ latex, $\mathrm{Tb}=$ tubercule, $\mathrm{Rz}=$ rhizome, $\mathrm{B}=$ bois, $\mathrm{Pl}=$ plante.

CM: Cameroun, CG : Congo, ST : Sao Tomé et principe, Td : Tchad, CA : Centrafrique, CD : Congo Démocratique , GE : Guinée Equatoriale, BU : Burundi, RW : Rwanda, GB : Gabon.

Tableau 2 (suite 5) : Résultats de l'inventaire des plantes tinctoriales d'Afrique centrale 


\begin{tabular}{|c|c|c|c|c|c|c|c|c|c|}
\hline Famille & Genre-espèce & $\begin{array}{l}\text { Nom } \\
\text { commun }\end{array}$ & État & $\begin{array}{l}\text { Partie } \\
\text { utilisée }\end{array}$ & $\begin{array}{l}\text { Type } \\
\text { croiss. }\end{array}$ & Répartition & $\begin{array}{l}\text { Principes } \\
\text { tinctoriaux }\end{array}$ & $\begin{array}{l}\text { Couleur de } \\
\text { l'extrait }\end{array}$ & Usages tinctoriaux \\
\hline \multirow{3}{*}{ Myrtaceae } & Psidium guayava L. ${ }^{*}$ & Goyavier & Sauv. & $\mathrm{Fr}, \mathrm{E}$ & Arbuste & $\begin{array}{l}\text { Toute la } \\
\text { région }\end{array}$ & - & $\begin{array}{l}\text { brun ou } \\
\text { pourpre }\end{array}$ & alimentaire \\
\hline & $\begin{array}{l}\text { Syzygium rowlandii } \\
\text { Sprague }\end{array}$ & - & Sauv. & $E$ & Arbre & $C A, C D$ & - & noire & Textile \\
\hline & Syzygium jambos & $\begin{array}{l}\text { Pomme - } \\
\text { Rose }\end{array}$ & $\begin{array}{l}\text { Sauv. } \\
\text { \&cult }\end{array}$ & $\mathrm{E}$ & Arbre & CG & - & Rouge & Non indiqué \\
\hline Nyctiginaceae & $\begin{array}{l}\text { Bougainvillea spectabilis } \\
\text { Willd* }\end{array}$ & Bougainvillier & $\begin{array}{l}\text { Sauv. } \\
\text { \&cult }\end{array}$ & $\mathrm{FI}$ & Arbuste & CG & $\begin{array}{l}\text { Anthocyanes, } \\
\text { quinones }\end{array}$ & Rouge violacé & Indicateur coloré \\
\hline Plombaginaceae & Plumbago zeylanica L. & Dentelaire & Sauv.\&cult & $\mathrm{Fl}$ & Arbuste & $\begin{array}{l}\text { Toute la } \\
\text { région }\end{array}$ & 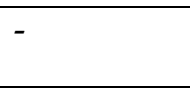 & noire & Non indiqué \\
\hline Phytolaccaceae & $\begin{array}{l}\text { Phytolacca dodecandran } \\
\text { L'Hér.* }^{*}\end{array}$ & phytolaque & Sauv. & $\mathrm{Fr}$ & Arbuste & $\begin{array}{l}\text { CG, GE, BU } \\
\mathrm{CM}, \mathrm{GB}, \mathrm{CD} \text {, } \\
\mathrm{CA}, \mathrm{RW},\end{array}$ & $\begin{array}{l}\text { Bétalaïnes, } \\
\text { anthocyanes }\end{array}$ & Pourpre/violet & Non indiqué \\
\hline Chrysobalanaceae & $\begin{array}{l}\text { Parina ricuratellifolia } \\
\text { Planch. Ex Benth. }\end{array}$ & & Sauv. & $E$ & Arbuste & $\begin{array}{l}\text { Toute la } \\
\text { région }\end{array}$ & - & Rouge & Non indiqué \\
\hline
\end{tabular}

$\mathrm{F}=$ feuille, $\mathrm{fl}=$ fleure, $\mathrm{Fr}=$ fruit, $\mathrm{E}=$ écorce, $\mathrm{Er}=$ écorce racine, $\mathrm{R}=$ racine, $\mathrm{G}=$ graine, $\mathrm{T}=$ tige, $\mathrm{Rmf}=$ rameaux feuillée, $\mathrm{Lt}=$ latex, $\mathrm{Tb}=$ tubercule, $\mathrm{Rz}=$ rhizome, $\mathrm{B}=$ bois, $\mathrm{Pl}=$ plante. CM: Cameroun, CG : Congo, ST : Sao Tomé et principe, Td : Tchad, CA : Centrafrique, CD : Congo Démocratique, GE : Guinée Equatoriale, BU : Burundi, RW : Rwanda, GB : Gabon. 
Tableau 2 (suite 6) : Résultats de l'inventaire des plantes tinctoriales d'Afrique centrale

\begin{tabular}{|c|c|c|c|c|c|c|c|c|c|}
\hline Famille & Genre-espèce & Nom commun & État & $\begin{array}{l}\text { Partie } \\
\text { utilisée }\end{array}$ & $\begin{array}{l}\text { Type } \\
\text { croiss. }\end{array}$ & Répartition & $\begin{array}{l}\text { Principes } \\
\text { tinctoriaux }\end{array}$ & $\begin{array}{l}\text { Couleur de } \\
\text { l'extrait }\end{array}$ & Usages tinctoriaux \\
\hline \multirow{16}{*}{$\begin{array}{l}\text { Fabaceae - } \\
\text { Faboïdeae }\end{array}$} & Baphia nitida Lood** & $\begin{array}{l}\text { Bois de Cam, } \\
\text { bois rouge }\end{array}$ & Sauv\&cult & $B, E, R$ & $\begin{array}{l}\text { Arbre / } \\
\text { arbuste }\end{array}$ & $\begin{array}{l}\text { GU, CM, ST, } \\
\text { GB }\end{array}$ & $\begin{array}{l}\text { isoflavonoïdes- } \\
\text { flavonoïdes }\end{array}$ & Rouge & Textile \\
\hline & Baphia laurifolia Bn. & - & Sauv. & $\mathrm{B}$ & Arbre & GB & - & Jaune & Non indiqué \\
\hline & $\begin{array}{l}\text { Flemingia grahamiana } \\
\text { Wight** }\end{array}$ & - & $\begin{array}{l}\text { Sauv. } \\
\text { \&cult }\end{array}$ & $\begin{array}{l}\mathrm{Fr} \\
\text { (glandes) }\end{array}$ & $\begin{array}{l}\text { Herbe / } \\
\text { arbuste }\end{array}$ & Toute la région & flavonoïdes & $\begin{array}{l}\text { Pourpre ou } \\
\text { brun }\end{array}$ & Cosmétique, textile, encre \\
\hline & $\begin{array}{l}\text { Indigofera arrecta Hochst. } \\
\text { Ex A.Rich }\end{array}$ & $\begin{array}{l}\text { Indigotier } \\
\text { chessé }\end{array}$ & $\begin{array}{l}\text { Sauv. } \\
\text { \&cult }\end{array}$ & Rmf & $\begin{array}{l}\text { Herbe I } \\
\text { arbuste }\end{array}$ & $\begin{array}{l}\text { CM, GB, CG, } \\
\text { CD,RW }\end{array}$ & Indigotine & Bleue & Textile \\
\hline & Indigofera coerulea Roxb** & Indigotier & $\begin{array}{l}\text { Sauv. } \\
\text { \&cult }\end{array}$ & Rmf & Herbe & Toute la région & Indigotine & Bleue & Textile \\
\hline & Macuna flagellipes Hook.f. & - & Sauv. & $F, T, E$ & Liane & $\mathrm{CD}, \mathrm{CA}, \mathrm{GB}$ & $\begin{array}{l}\text { Hétérosides } \\
\text { cyanogéniques }\end{array}$ & $\begin{array}{l}\text { Noire, bleu- } \\
\text { noire }\end{array}$ & Textile, poterie \\
\hline & Macuna poggei Taub** & $\begin{array}{l}\text { Macuna de } \\
\text { pogge }\end{array}$ & Sauv. & $\mathrm{F}, \mathrm{T}$ & Liane & Toute la région & - & Noire & Encre \\
\hline & $\begin{array}{l}\text { Macuna sloanei Fawc } \\
\text { \&rendle }\end{array}$ & $\begin{array}{l}\text { OEil de } \\
\text { bourrique }\end{array}$ & $\begin{array}{l}\text { Sauv. } \\
\text { \&cult }\end{array}$ & $\mathrm{PI}$ & Liane & CD & - & Noire & Textile, peinture \\
\hline & $\begin{array}{l}\text { Philenoptera cyanescens } \\
\text { Roberty }\end{array}$ & $\begin{array}{l}\text { Gara, liane- } \\
\text { indigo }\end{array}$ & $\begin{array}{l}\text { Sauv. } \\
\text { \&cult }\end{array}$ & Rmf & $\begin{array}{l}\text { Arbuste } \\
\text { / liane }\end{array}$ & $\mathrm{CM}, \mathrm{GE}$ & $\begin{array}{l}\text { Indigotine + } \\
\text { polyphénols }\end{array}$ & indigo & Textile \\
\hline & $\begin{array}{l}\text { Philenoptera laxiflora (Guill. } \\
\text { \&Perr.) Roberty }\end{array}$ & Savonette & Sauv. & Rmf & Arbuste & $\begin{array}{l}\text { Td, CM, CD, } \\
\mathrm{CA}\end{array}$ & - & Teinture indigo & Textile \\
\hline & $\begin{array}{l}\text { Pterocarpus angolensis } \\
\text { DC. }\end{array}$ & $\begin{array}{l}\text { Mninga } \\
\text { (swhahili) }\end{array}$ & $\begin{array}{l}\text { Sauv. } \\
\text { \&cult }\end{array}$ & $R, B, E$ & Arbuste & CD & isoflavonoïdes & $\begin{array}{l}\text { Rouge } \\
\text { brunâtre }\end{array}$ & Non indiqué \\
\hline & Pterocarpus soyauxii taub** & $\begin{array}{l}\text { Padouk } \\
\text { d'Afrique }\end{array}$ & Sauv. & $R, B, E$ & Arbuste & $\begin{array}{l}\text { CG, CM, CA, } \\
\text { GE, GB, CD }\end{array}$ & biflavonoïdes & Rouge & Cosmétique, textile \\
\hline & $\begin{array}{l}\text { Physostigma venenosum } \\
\text { Balf. }\end{array}$ & $\begin{array}{l}\text { Fève de } \\
\text { calabar }\end{array}$ & Sauv. & $F$ & Liane & $\mathrm{CD}, \mathrm{GB}$ & - & Noire & Vannerie \\
\hline & $\begin{array}{l}\text { Lonchocarpus sericeus H.B } \\
\& \mathrm{~K}\end{array}$ & Membrurier & Sauv. & $F, G$ & Arbre & GB, CD & - & $\begin{array}{l}\text { Verte/Jaune- } \\
\text { orangé }\end{array}$ & Textile, vannerie \\
\hline & Indigofera tinctoriaL. & $\begin{array}{l}\text { Indigotier } \\
\text { tinctorial }\end{array}$ & $\begin{array}{l}\text { Sauv. } \\
\text { \&cult }\end{array}$ & Rmf & $\begin{array}{l}\text { Herbe / } \\
\text { arbuste }\end{array}$ & $\begin{array}{l}\text { Td, CA, CM, } \\
\text { GB, ST }\end{array}$ & Indoxyle & Bleu & Textile \\
\hline & Arachishy pogeae & Arachide & Cult. & G & Herbe & Toute la région & & Brun & Non indiqué \\
\hline
\end{tabular}

$\mathrm{F}=$ feuille, $\mathrm{fl}=$ fleure, $\mathrm{Fr}=$ fruit, $\mathrm{E}=$ écorce, $\mathrm{Er}=$ écorce racine, $\mathrm{R}=$ racine, $\mathrm{G}=$ graine, $\mathrm{T}=$ tige, $\mathrm{Rmf}=$ rameaux feuillée, $\mathrm{Lt}=$ latex, $\mathrm{Tb}=$ tubercule, $\mathrm{Rz}=$ rhizome, $\mathrm{B}=$ bois, $\mathrm{PI}=$ plante . CM: Cameroun, CG : Congo, ST : Sao Tomé et principe, Td : Tchad, CA : Centrafrique, CD : Congo Démocratique, GE : Guinée Equatoriale, BU : Burundi, RW : Rwanda, GB : Gabon. 
Tableau 2 (suite 7) : Résultats de l'inventaire des plantes tinctoriales d'Afrique centrale

\begin{tabular}{|c|c|c|c|c|c|c|c|c|c|}
\hline Famille & Genre-espèce & Nom commun & État & $\begin{array}{l}\text { Partie } \\
\text { utilisée }\end{array}$ & $\begin{array}{l}\text { Type } \\
\text { croiss. }\end{array}$ & Répartition & $\begin{array}{l}\text { Principes } \\
\text { tinctoriaux }\end{array}$ & $\begin{array}{l}\text { Couleur de } \\
\text { l'extrait }\end{array}$ & Usages tinctoriaux \\
\hline \multirow{13}{*}{ Rubiaceae } & $\begin{array}{l}\text { Adenorandia kalbreyeri } \\
\text { Robbr. }\end{array}$ & - & Sauv. & $\mathrm{Fr}$ & $\begin{array}{l}\text { Arbre/ } \\
\text { liane }\end{array}$ & $C D$ & - & Bleue & Cosmétique, tatouage \\
\hline & $\begin{array}{l}\text { Aoranthean nulata } \\
\text { (K.Schum) Somers }\end{array}$ & - & Sauv. & $\mathrm{R}$ & Arbre & GB, CG & - & Noir & Cosmétique, tatouage \\
\hline & $\begin{array}{l}\text { Craterispermum } \\
\text { schweinfurthii Hiern** }\end{array}$ & - & Sauv. & $B, E, F$ & $\begin{array}{l}\text { Arbre / } \\
\text { arbuste }\end{array}$ & Toute la région & - & Jaune et brune & Cosmétique, textile \\
\hline & $\begin{array}{l}\text { Cremas poratriflora (Thonn.) } \\
\text { K. Schum }\end{array}$ & - & Sauv. & Fr, G & $\begin{array}{l}\text { Arbre / } \\
\text { arbuste }\end{array}$ & $\mathrm{CD}, \mathrm{GB}$ & - & Bleu-noire & cosmétique \\
\hline & Morinda lucida Benth** & Arbre à soufre & $\begin{array}{l}\text { Sauv. } \\
\text { \&cult }\end{array}$ & $F, B, R$ & $\begin{array}{l}\text { Arbre / } \\
\text { arbuste }\end{array}$ & Toute la région & Anthraquinone & Jaune, rouge & textile, \\
\hline & Randia Le testui Pellegr. & & Sauv. & $\mathrm{Fr}$ & Liane & GB & - & Noire & Non rapporté \\
\hline & $\begin{array}{l}\text { Randia malleifera } \\
\text { Benth\&Hook. }\end{array}$ & & Sauv. & $\mathrm{Fr}$ & Arbuste & GB & - & Noire & Tatouage \\
\hline & $\begin{array}{l}\text { Pauridiantha rubens (Benth.) } \\
\text { Bremek. }\end{array}$ & - & Sauv. & $\mathrm{F}$ & $\begin{array}{l}\text { Arbre / } \\
\text { arbuste }\end{array}$ & $\begin{array}{l}\mathrm{CA}, \mathrm{CA}, \mathrm{GE}, \\
\mathrm{GB}, \mathrm{CD}\end{array}$ & - & rouge & Non rapporté \\
\hline & $\begin{array}{l}\text { Psilanthuse bractiolatus } \\
\text { Hiern }\end{array}$ & - & Sauv. & Fr, G & $\begin{array}{l}\text { Arbre / } \\
\text { arbuste }\end{array}$ & $\mathrm{GE}, \mathrm{CA}$ & - & Noire & Tatouage \\
\hline & $\begin{array}{l}\text { Psychotria psychotriodes } \\
\text { (DC.) }\end{array}$ & $\begin{array}{l}\text { Azier, café } \\
\text { marron }\end{array}$ & Sauv. & $E, F$ & Arbuste & $\mathrm{GB}, \mathrm{RDC}$ & - & rouge & Textile \\
\hline & Rothmannia iongiflora Salisb & - & Sauv. & $\begin{array}{l}\text { Fr, F, G, } \\
\mathrm{Fl}\end{array}$ & $\begin{array}{l}\text { Arbre / } \\
\text { arbuste }\end{array}$ & $\begin{array}{l}\mathrm{CA}, \mathrm{CA}, \mathrm{RW} \\
\mathrm{GB}, \mathrm{CG}, \mathrm{CD}\end{array}$ & - & Noir- bleutée & Cosmétique, encre \\
\hline & Gardenia kalbreyeri Hiern & & Sauv. & Sève & Liane & $\mathrm{GB}, \mathrm{GE}$ & - & Noire & Non indiqué \\
\hline & $\begin{array}{l}\text { Rothmannia whitfieldii } \\
\text { Dandy }\end{array}$ & - & Sauv. & Fr, G & $\begin{array}{l}\text { Arbre / } \\
\text { arbuste }\end{array}$ & Toute la région & - & Noir bleuté & Cosmétique, encre \\
\hline \multirow[t]{2}{*}{ Olacaceae } & Ximenia Americana L. & & Sauv. & & Arbuste & GE, ST, CM & - & & Non indiqué \\
\hline & $\begin{array}{l}\text { Strombosia grandifolia } \\
\text { Hook. F. }\end{array}$ & & Sauv. & sève & Arbuste & GB, CG & - & Noire & Poterie \\
\hline
\end{tabular}

$\mathrm{F}=$ feuille, $\mathrm{fl}=$ fleure, $\mathrm{Fr}=$ fruit, $\mathrm{E}=$ écorce, $\mathrm{Er}=$ écorce racine, $\mathrm{R}=$ racine, $\mathrm{G}=$ graine, $\mathrm{T}=$ tige, $\mathrm{Rmf}=$ rameaux feuillée, $\mathrm{Lt}=$ latex, $\mathrm{Tb}=$ tubercule, $\mathrm{Rz}=$ rhizome, $\mathrm{B}=$ bois, $\mathrm{Pl}=$ plante CM: Cameroun, CG : Congo, ST : Sao Tomé et principe, Td : Tchad, CA : Centrafrique, CD : Congo Démocratique, GE : Guinée Equatoriale, BU : Burundi, RW : Rwanda, GB : Gabon. 
Tableau 2 (suite 8): Résultats de l'inventaire des plantes tinctoriales d'Afrique centrale

\begin{tabular}{|c|c|c|c|c|c|c|c|c|c|}
\hline Famille & Genre-espèce & Nom commun & Etat & $\begin{array}{l}\text { Partie } \\
\text { utilisée }\end{array}$ & $\begin{array}{l}\text { Type } \\
\text { croiss. }\end{array}$ & Répartition & $\begin{array}{l}\text { Principes } \\
\text { tinctoriaux }\end{array}$ & $\begin{array}{l}\text { Couleur de } \\
\text { l'extrait }\end{array}$ & Usages tinctoriaux \\
\hline \multirow{7}{*}{ Rubiaceae } & Rubia coradifolia L. & Garance indie & Sauv. & $\mathrm{R}, \mathrm{Fr}$ & Herbe & $\mathrm{CD}, \mathrm{RW}, \mathrm{BU}$ & anthraquinones & Rouge, Violet & Textile \\
\hline & Sabicea affinis De Wild. & & Sauv. & $\mathrm{Fr}$ & Arbuste & GB & & Rouge & Encre \\
\hline & $\begin{array}{l}\text { Sarcocephalus esculentus } \\
\text { Afzel }\end{array}$ & & Sauv. & $\mathrm{R}$ & Arbuste & $\mathrm{GB}, \mathrm{CG}, \mathrm{CD}$ & - & Jeune foncé & comestique \\
\hline & Coffea spp* & caféier & Cult. & $\mathrm{F}$ & Arbuste & $\begin{array}{l}\mathrm{CD}, \mathrm{RW}, \mathrm{GB} \\
\mathrm{CM}, \mathrm{CG}\end{array}$ & - & Marron & alimentaire \\
\hline & Morinda citrifolia L. ${ }^{*}$ & Noni & Sauv. & - & Arbuste & Toute la région & - & $\begin{array}{l}\text { Jaune, rouge } \\
\text { ou pourpre }\end{array}$ & $\begin{array}{l}\text { Alimentaire } \\
\text { cosmétique }\end{array}$ \\
\hline & $\begin{array}{l}\text { Crossopteryx febrifuga } \\
\text { (Afzel.ex.G.Don) Benth. }\end{array}$ & & Sauv. & & & Toute la région & - & & Non indiqué \\
\hline & Nauclea latifolia Sm. & & Sauv. & $E, R, B$ & Liane & Toute la région & - & Jaune & Non indiqué \\
\hline \multirow[t]{2}{*}{ Rhizophoraceae } & Poga oleosa Pierre & $\begin{array}{l}\text { Erable } \\
\text { d'Afrique }\end{array}$ & Sauv. & $\mathrm{E}$ & Arbre & $\mathrm{GB}, \mathrm{CG}$ & Tanins & Noire & Textile \\
\hline & $\begin{array}{l}\text { Rhizophora racemosa } \\
\text { G.F.V.Mey }\end{array}$ & $\begin{array}{l}\text { Manglier ou } \\
\text { palétuvier } \\
\text { rouge }\end{array}$ & Sauv. & $E$ & Arbre & $\begin{array}{l}\text { GB, CG, GU, } \\
\mathrm{CM}\end{array}$ & Tanins & Rouge, noire & Artisanat \\
\hline \multirow{3}{*}{ Scrophulariaceae } & $\begin{array}{l}\text { Alectra sessiliflora (Vahl) } \\
\text { Kuntze }\end{array}$ & - & Sauv. & $\mathrm{Fl} ; \mathrm{R}$ & Herbe & Toute la région & - & Jauned'or & Textile \\
\hline & $\begin{array}{l}\text { Buchnera hispida Buch.- } \\
\text { ham. Ex D.Don }\end{array}$ & - & Sauv. & $\mathrm{Pl}$ & Herbe & Toute la région & - & Noir & Non rapporté \\
\hline & $\begin{array}{l}\text { Strigages nerioides (Willd.) } \\
\text { Vatke }\end{array}$ & Striga & Sauv. & $\mathrm{R}, \mathrm{FI}$ & Herbe & Toute la région & Glucosidesiridoïdes & Bleu-noir & Cosmétique, textile \\
\hline Simaroubaceae & Simarouba glauca DC & Bois amer & Sauv. & $E$ & Arbre & $\mathrm{CM}, \mathrm{CD}, \mathrm{CA}$ & & Jaune & Non indiqué \\
\hline \multirow[t]{2}{*}{ Solanaceae } & $\begin{array}{l}\text { Lycopersicum esculentum } \\
\text { P. Miller ** }\end{array}$ & tomate & Cult. & FR & plante & Toute la région & caroténoïdes & Rouge & Alimentaire \\
\hline & CapsicumfrutescensL. * & Piment & Sauv.\&cult & $\mathrm{Fr}$ & Arbuste & CG, GE, CD & - & Rouge & Alimentaire \\
\hline Asclépiadaceae & $\begin{array}{l}\text { Cryptolepis sanguinolenta } \\
\text { Schltr. }\end{array}$ & - & Sauv. & $\mathrm{R}$ & Arbre & $\begin{array}{l}\mathrm{CM}, \mathrm{CA}, \mathrm{CG}, \\
\mathrm{CD}\end{array}$ & cryptolépine & jaune & textile \\
\hline Gramineae & Chevalierella Congolensis A. & - & Sauv. & - & Arbuste & CG & - & jaune & Non indiqué \\
\hline
\end{tabular}

$\mathrm{F}=$ feuille, $\mathrm{fl}=$ fleure, $\mathrm{Fr}=$ fruit, $\mathrm{E}=$ écorce, $\mathrm{Er}=$ écorce racine, $\mathrm{R}=$ racine, $\mathrm{G}=$ graine, $\mathrm{T}=$ tige, $\mathrm{Rmf}=$ rameaux feuillée, $\mathrm{Lt}=$ latex, $\mathrm{Tb}=$ tubercule, $\mathrm{Rz}=$ rhizome, $\mathrm{B}=$ bois, $\mathrm{Pl}=$ plante.

CM: Cameroun, CG : Congo, ST : Sao Tomé et principe, Td : Tchad, CA : Centrafrique, CD : Congo Démocratique, GE : Guinée Equatoriale, BU : Burundi, RW : Rwanda, GB : Gabon. 
Tableau 2 (suite 9) : Résultats de l'inventaire des plantes tinctoriales d'Afrique centrale

\begin{tabular}{|c|c|c|c|c|c|c|c|c|c|}
\hline Famille & Genre-espèce & Nom commun & État & $\begin{array}{l}\text { Partie } \\
\text { utilisée }\end{array}$ & $\begin{array}{l}\text { Type } \\
\text { croiss. }\end{array}$ & Répartition & $\begin{array}{l}\text { Principes } \\
\text { tinctoriaux }\end{array}$ & $\begin{array}{l}\text { Couleur de } \\
\text { l'extrait }\end{array}$ & Usages tinctoriaux \\
\hline \multirow{5}{*}{ Malvaceae } & Hibiscus sabdariffa L. ** & $\begin{array}{l}\text { Oseille de } \\
\text { guinée }\end{array}$ & $\begin{array}{l}\text { Sauv. } \\
\text { \&cult }\end{array}$ & $\mathrm{F}, \mathrm{Fr}$ & Herbe & Toute la région & Anthocyanes & rouge & Cosmétique, alimentaire \\
\hline & Desplatsia subericarpa Bocq. & & Sauv. & $\mathrm{Fr}$ & Arbuste & GB, CG, GE & - & Noire & Non indiqué \\
\hline & Grewia coriacea Mast * & Arbre à briquet & Sauv. & $\mathrm{Fr}$ & Arbre & $\begin{array}{l}\mathrm{CM}, \mathrm{CD}, \mathrm{CG}, \\
\mathrm{GB}\end{array}$ & Anthocyanidols & Rouge - violacé & Alimentaire \\
\hline & Grewia bicolor * & & Sauv. & $\mathrm{Fr}$ & $\begin{array}{l}\text { Arbre/ } \\
\text { arbuste }\end{array}$ & CG, GB & - & Rouge - violacé & Alimentaire \\
\hline & Théobroma cacao L. ** & Cacaoyer & $\begin{array}{l}\text { Sauv. } \\
\text { \&cult }\end{array}$ & $\mathrm{Fr}$ & Arbuste & $\begin{array}{l}\text { CG, GE, GB, } \\
\text { CD }\end{array}$ & - & Marron & Alimentaire, cosmetique \\
\hline \multirow{3}{*}{ Verbanaceae } & Tectona Grandis L. ** & Teck & $\begin{array}{l}\text { Sauv. } \\
\text { \&cult }\end{array}$ & $\mathrm{F}$ & Arbre & $\begin{array}{l}\mathrm{CG}, \mathrm{GE}, \mathrm{GB}, \\
\mathrm{CM}, \mathrm{CD}\end{array}$ & - & Rouge & Cosmétique, encre, textile \\
\hline & $\begin{array}{l}\text { Clerodendron Scandens } \\
\text { P.Beauv. }\end{array}$ & - & $\begin{array}{l}\text { Sauv. } \\
\text { \&cult }\end{array}$ & $\mathrm{F}$ & Arbuste & CG, GB & - & Noire & Non indiqué \\
\hline & $\begin{array}{l}\text { Clerodendron splendens } \\
\text { Don. }\end{array}$ & - & Sauv. & $\mathrm{F}$ & Liane & CG, GB, CD & - & Noire & Non indiqué \\
\hline Palmaceae & Eleacis guineensis Jacq * & Palmier à huile & $\begin{array}{l}\text { Sauv. } \\
\text { \&cult }\end{array}$ & $\mathrm{Fr}$ & - & Toute la région & - & Jaune-orangé & Alimentaire, cosmetique \\
\hline Theacea & $\begin{array}{l}\text { Camellia simensis (L.) O. } \\
\text { Kuntze* }^{* *}\end{array}$ & Théier & Cult. & $\mathrm{F}$ & Arbuste & $\begin{array}{l}\text { CM, CD, GB, } \\
C G, G E\end{array}$ & - & $\begin{array}{l}\text { Vert ou noir, } \\
\text { jaune pâle }\end{array}$ & Boisson \\
\hline Meliaceae & Carapa procera DC & & Sauv. & G & Arbre & $\begin{array}{l}\mathrm{CM}, \mathrm{CD}, \mathrm{GB}, \\
\mathrm{CG}\end{array}$ & - & Rougeâtre & Cosmétique \\
\hline Zingiberaceae & Curcuma longa L. * & Curcuma & Cult. & Rz & Herbe & Toute la région & $\begin{array}{l}\text { caroténoïdes : } \\
\text { curcuminoïdes. }\end{array}$ & Jaune d'or & $\begin{array}{l}\text { Alimentaire, Cosmétique et } \\
\text { textile }\end{array}$ \\
\hline \multirow{3}{*}{ Clusiaceae } & Garcinia Kola Helkel* & - & Sauv. & $\mathrm{Fr}$ & & $\begin{array}{l}\text { CG, GB, GE, } \\
\text { CM, CD }\end{array}$ & - & Orange & Non indiqué \\
\hline & Symphonia globuliferaL. * & - & Sauv. & E, Lt & Arbre & CG & - & jaune & Non indiqué \\
\hline & Garcia huilensis* & - & Sauv. & $R$ & Arbuste & CG, GB & - & jaune & Non indiqué \\
\hline Cactaceae & $\begin{array}{l}\text { Hylocerussp. Var orejona, } \\
\text { Lisa, Rosa (A. Berger) }\end{array}$ & fruit du dragon & $\begin{array}{l}\text { Sauv. } \\
\text { \&cult. }\end{array}$ & $\mathrm{Fr}$ & Arbre & CM, ST, GE & Betalaïne & orange & Cosmétique, alimentaire \\
\hline
\end{tabular}

$F=$ feuille,$f l=$ fleure, $F r=$ fruit, $E=$ écorce, $E r=$ écorce racine,$R=$ racine,$G=$ graine,$T=$ tige, $R m f=$ rameaux feuillée, $L t=$ latex, $T b=$ tubercule, $R z=$ rhizome, $B=$ bois, $P I=$ plante.

CM: Cameroun, CG : Congo, ST : Sao Tomé et principe, Td : Tchad, CA : Centrafrique, CD : Congo Démocratique, GE : Guinée Equatoriale, BU : Burundi, RW : Rwanda, GB : Gabon. 